\title{
Some Results on Fixed Points of Nonlinear Contraction in Metric Space
}

\author{
Dr. Durdana Lateef \\ Department of Mathematics, College of Science, Taibah University, \\ Al-Madinah Al-Munawwarah, 41411, Kingdom of Saudi Arabia.
}

\begin{abstract}
In this paper, I have generalized the result of Sayyed and Sayyed [17] in metric spaces by replacing the containment condition and giving the shorter proof than of the authors in the main result.
\end{abstract}

Keywords and Phrases: Common fixed point, Compatible mapping, Property (E.A.), Common property (E.A.), Occasionally weakly compatible maps, Coincidence points.

\section{INTRODUCTION}

Aamri and Moutawakil [1] introduced the concept of property (E.A.) which was perhaps inspired by the condition of compatibility introduced by Jungck [11] and further Imdad and Ali[10] extended this result . Recently Babu and Alemayehu [7, 8,9] proved common fixed point theorem for occasionally weakly compatible maps satisfying property (E.A.) using an inequality involving quadratic terms. Aliouche[4] proved a common fixed point theorem of Gregus type weakly compatible mappings satisfying generalized contractive conditions.

Abbas [2] established a common fixed point for Lipschitzian mapping satisfying rational contractive conditions. Murty et.al [15] proved fixed points of nonlinear contraction in metric space.

\section{Preliminaries}

Throughout this paper $(X, d)$ is a metric space which is denoted by $X$.

Definition 2.1: [Jungck and Rhoades [13]]. Let $A$ and $S$ be selfmaps of a set $X$. If $A u=S u=\omega$ (say), $\omega \in \mathrm{X}$, for some $u$ in $X$, then $u$ is called a coincidence point of $A$ and $S$ and the set of coincidence points of $A$ and $S$ is denoted by $C(A, S)$, and $\omega$ is called a point of coincidence of $A$ and $S$.

Definition 2.2: Let A, B, S and T be self maps of a set $X$. If $u \in C(A, S)$ and $v \in C(B, T)$ for some $u, v \in X$ and $A u=S u=B v=T v=z$ (say), then $z$ is called a common point of coincidence of the pairs $(A . S)$ and $(B . T)$.

Definition 2.3: The pair $(A, S)$ is said to be

(I) Satisfy property (E.A.) [1] if there exists a sequence $\left\{x_{n}\right\}$ in $X$ such that $\lim _{n \rightarrow \infty} A x_{n}=\lim _{n \rightarrow \infty} S x_{n}=t$ for some $t$ in $X$.

(II) Copatible [11] if $\lim _{n \rightarrow \infty} d\left(A S x_{n}, S A x_{n}\right)=0$, for some $t$ in $X$ whenever $\left\{x_{n}\right\}$ is a sequence in $X$ such that $\lim _{n \rightarrow \infty} A x_{n}=\lim _{n \rightarrow \infty} S x_{n}=t$.

(III) Weakly compatible [12], if they commute at their coincidence point.

(IV) Occasionally weakly compatible (owc) $[3,5,6]$ if $A S x=S A x$ for some $x \in C(A, S)$.

\section{Remark 2.4}

(I) Every compatible pair is weakly compatible but its converse need not be true [12]. 
(II) Weak compatibility and property (E. A.) are independent of each other [16].

(III) Every weakly compatible pair is occasionally weakly compatible but its converse need not be true [11].

(IV) Occasionally weakly compatible and property (E.A.) are independent of each other [8].

Definition 2.5: [14] Let $(X, d)$ be a metric space and $A, B, S$ and $T$ be four selfmaps on X. The pairs $(A, S)$ and $(B, T)$ are said to satisfy common property (E.A.) if there exists two sequences $\left\{x_{n}\right\}$ and $\left\{y_{n}\right\}$ in $\mathrm{X}$ such that $\lim _{n \rightarrow \infty} A x_{n}=\lim _{n \rightarrow \infty} S x_{n}=t=\lim _{n \rightarrow \infty} B y_{n}=\lim _{n \rightarrow \infty} T y_{n}$ for some $\mathrm{t}$ in $\mathrm{X}$.

Remark 2.6: Let $A, B, S$ and $T$ be self maps of a set X. If the pairs $(A, S)$ and $(B, T)$ have common point of coincidence in $X$ then $C(A, S) \neq \phi$ and $C(B, T) \neq \phi$. But converse is not true.

Example 2.7: Let $X=[0, \infty)$ with usual metric and $A, B, S$ and $T$ self maps on $x$ and defined by $A x=1-x^{2} ; s x=1-x ; B x=\frac{1}{2}+x^{2} ; T x=\frac{1+x}{2}$ for all $x \in X$.

It is easy to observe that $C(A, S)=\{0,1\}$ and $C(B, T)=\left\{0, \frac{1}{2}\right\}$ but the pairs $(A, S)$ and $(B, T)$ not having common point of coincidence.

Remark 2.8: The converse of the remark 2.6 is true provided it satisfies inequality (3.1). This is given as in proposition 3.1 in section III.

Preposition 2.9: [2] Let $A$ and $S$ be two self maps of a set $X$ and the pair $(A, S)$ is satisfies occasionally weakly compatible (owc) condition. If the pairs $(A, S)$ have unique point of coincidence $A x=S x=z$ then $z$ is the unique common fixed point of $A$ and $S$.

Proof: To be given $A x=S x=\{z\}$ (say) for any $x \in C(A, S)$

Since the pair $(A, S)$ satisfies the property owc, therefore

$$
A z=A S x=S A x=S z \text { implies that } z \in C(A, S)
$$

From (2.1), we get $A z=S z=z$. Hence proposition follows.

In 1996, Tas et al. [17] proved the following.

Theorem 2.10: Let $A, B, S$ and $T$ be selfmaps of a complete metric space $(X, d)$ such that $A(X) \subseteq T(X)$ and $B(X) \subseteq S(X)$ and satisfying the inequality.

$$
\begin{aligned}
{[d(A x, B y)]^{2} \leq } & C_{1} \max \left\{[d(S x, A x)]^{2},[d(T y, B y)]^{2},[d(S x, T y)]^{2}\right\} \\
& +C_{2} \max \{d(S x, A x) d(S x, B y), d(T y, A x) d(T y, B y)\} \\
& +C_{3} d(S x, B y) d(T y, A x)
\end{aligned}
$$

for all $x, y \in x$, where $C_{1}+C_{3}, C_{2}, C_{3} \geq 0, C_{1}+2 C_{2}<1, C_{1}+C_{3}<1$. Further, assume that the pairs $(A, S)$ and $(B, T)$ are compatible onX. If one of the mappings $A, B, S$ and $T$ is continuous then $A, B, S$ and $T$ have a unique common fixed point in $X$.

\section{Main Results}

Proposition 3.1. Let $A, B, S$ and $T$ be self maps of a metric space $(X, d)$ and satisfying the inequality.

$$
\begin{gathered}
\mathrm{d}(\mathrm{Ax}, \mathrm{By}) \leq \mathrm{k} \max \left\{\frac{d(S x, A x)[1+d(T y, B y)]}{1+d(S x, t y)}, \frac{d(T y, B y)[1+d(S x, A x)]}{1+d(S x, T y)}, \frac{d(S x, A x)[1+d(S x, T y)]}{1+d(T y, B y)}\right. \\
, \frac{d(S x, T y)[1+d(S x, A x)]}{1+d(T y, B y)}, \frac{1}{2}[\mathrm{~d}(\mathrm{Sx}, \mathrm{Ax})+\mathrm{d}(\mathrm{Ty}, \mathrm{By})] \\
\left., \frac{1}{2}[\mathrm{~d}(\mathrm{Sx}, \mathrm{By})+\mathrm{d}(\mathrm{Ty}, \mathrm{Ax})], \mathrm{d}(\mathrm{sx}, \mathrm{Ty})\right\}
\end{gathered}
$$


for all $x, y \in x$, where $\mathrm{k} \geq 0$ and $\mathrm{k}<1$. Then the pairs $(A, S)$ and $(B, T)$ have common point of coincidence in $\mathrm{X}$ if and only if $C(A, S) \neq \phi$ and $C(B, T) \neq \phi$.

Prof: If part: It is trivial

Only if part: Assume $C(A, S) \neq \phi$ and $C(B, T) \neq \phi$.

Then there is a $u \varepsilon C(A, S)$ and $v_{\varepsilon} C(B, T)$ such that

$$
\begin{aligned}
& A u=S u=p \text { (say) } \\
& B v=T v=q \quad \text { (say) }
\end{aligned}
$$

on taking $x=u$ and $y=v$ in (3.1), we get

$$
\begin{aligned}
\mathrm{d}(\mathrm{Au}, \mathrm{Bv}) \leq \mathrm{k} \max \left\{\frac{d(s u, A u)[1+d(t v, B v)]}{1+d(S u, t v)}, \frac{d(T v, B v)[1+d(S u, A u)]}{1+d(S u, T v)}, \frac{d(S u, A u)[1+d(S u, T v)]}{1+d(T v, B v)},\right. \\
\frac{d(S u, T v)[1+d(S u, A u)]}{1+d(T v, B v)}, \frac{1}{2}[d(S u, A u)+d(T v, B v)], \\
\left.\frac{1}{2}[d(S u, B v)+d(T v, A u)], \mathrm{d}(\mathrm{Su}, \mathrm{Tv})\right\}
\end{aligned}
$$

Using (3.2) and (3.3), we get

$$
\mathrm{d}(\mathrm{p}, \mathrm{q}) \leq \mathrm{k} \mathrm{d}(\mathrm{p}, \mathrm{q}) \text {, a contradiction. Thus } p=q
$$

Therefore $A, B, S$ and Thave common point of coincidence in X.

In The proposition (2.1) of Babu et al. [9], we can obtain some more conclusions of in their paper. Therefore our result improves and strengthen proposition 3.1 and subsequent theorems in metric spaces.

Proposition 3.2: Let $A, B, S$ and $T$ be four self maps of a metric space $(X, d)$ satisfying the inequality (3.1). Suppose that either

(i) $B(X) \subseteq S(X)$, the pair $(B, T)$ satisfies property (E.A.) and $T(X)$ is a closed subspace of $X$; or

(ii) $A(X) \subseteq T(X)$, the pair $(A, S)$ satisfies property $(E . A)$ and $S(X)$ is a closed subspace of $\mathrm{X}$ holds.

Then the pair $(A, S)$ and $(B, T)$ are satisfies the common property (E.A), also both the pairs $(A, S)$ and $(B, T)$ have common point of coincidence in $X$.

We have shorten the proof of theorem 2.2 of [9] by relaxing many lines:

Theorem 3.3: (Improved version of theorem 2.2of [9])

Let $A, B, S$ and $T$ are satisfying all the conditions given in proposition 3.2 with the following additional assumption.

The pairs $(A, S)$ and $(B, T)$ are owc on $X$.

Then $A, B, S$ and $T$ have a unique common fixed point in $X$.

Proof: By proposition 3.2 the pairs $(A, S)$ and $(B, T)$ have common point of coincidence. Therefore there is $u \in C(A, S)$ and $v \in C(B, T)$ such that

$$
A u=S u=z \text { (say) }=B v=T v
$$

Now, we show that $z$ is unique common point of coincidence of the pairs $(A, S)$ and $(B, T)$.

Let if possible $z^{\prime}$ is another point of coincidence of $A, B, S$ and $T$. Then there is $u^{\prime} \varepsilon C(A, S)$ and $v^{\prime} \varepsilon C(B, T)$ such that

$$
A u^{\prime}=S u^{\prime}=z^{\prime}(\text { say })=B v^{\prime}=T v^{\prime}
$$


Putting $x=u$ and $y=v^{\prime}$ in inequality (3.1), we have

$$
\begin{aligned}
& \mathrm{d}\left(\mathrm{Au}, \mathrm{Bv}^{\prime}\right) \leq \mathrm{k} \max \left\{\frac{d(S u, A u)\left[1+d\left(T v^{\prime} \cdot B v\right)\right]}{1+d(S u, T v)}, \frac{d\left(T v^{\prime}, B v\right)[1+d(S u, A u)]}{1+d(S u, T v)}\right. \\
& , \frac{d(S u, A u)[1+d(S u, T v)]}{1+d\left(T v^{\prime}, B v\right)}, \frac{d(S u, T v)[1+d(S u, A u)]}{1+d\left(T v^{\prime}, B v\right)}, \frac{1}{2}[d(S u, A u)+ \\
& \left.d\left(T v^{\prime}, B v^{\prime}\right)\right], \quad \frac{1}{2}\left[d\left(S u, B v^{\prime}\right)+\right. \\
& \left.\left.d\left(T v^{\prime}, A u\right)\right], \mathrm{d}\left(\mathrm{Su}, T v^{\prime}\right)\right\}
\end{aligned}
$$

Now using (3.4) and (3.5), we get

$\mathrm{d}\left(\mathrm{z}, \mathrm{z}^{\prime}\right) \leq \mathrm{kd}\left(\mathrm{z}, \mathrm{z}^{\prime}\right)$, and arrive at a contradiction. Hence $z=z^{\prime}$ and we have

$C(A, S)=\{z\}=C(B, T)$. By proposition $2.9, z$ is the unique common fixed point of $A, B, S$ and $T$ in $X$.

Remark 3.4: Proposition 2.5 of [9] and theorem 2.6 of [9] are remain true, if we replace completeness of $S(X)$ and $T(X)$ by the completeness of $S(X) \cap T(X)$ in X. For this we have given an example 2.7 in the following manner without proof.

Now we rewriting the proposition 2.5 and theorem 2.6 of [9]

Proposition 3.5: Let $A, B, S$ and $T$ be four self maps of a metric. Space $(X, d)$ satisfying the inequality (3.1) of proposition 3.1. Suppose that $(A, S)$ and $(B, T)$ satisfy a common property $(E . A)$ and $S(X) \cap T(X)$ are closed subset of $X$, then $A, B, S$ and $T$ have unique common point of coincidence. Theorem 3.6. In addition to the above proposition 3.5 on $A, B, S$ and $T$, if both the pairs $(A, S)$ and $(B, T)$ are owc mapson $X$, then the point of coincidence is a unique common point of $A, B, S$ and $T$.

\section{REFERENCES}

[1] Aamri, M., El Moutawakil, D., Some new common fixed point theorems under strict contractive conditions, J. Math. Anal. Appl., 270(2002), 181-188.

[2] Abbas, M., Common fixed point for Lipschitzian mapping satisfying rational contractive conditions, Italian journal of pure and Applied Mathematics, 27(2010), 141-146.

[3] Abbas, M., Rhoades, B.E., Common fixed point theorems for occasionally weakly compatible mappings satisfying a general contractive condition, Math. Commun., 13(2008), 295-301.

[4] Aliouche, A., Common fixed point theorems of Gregus type weakly compatible mappings satisfying generalized contractive conditions, J. Math. Anal. Appl., 341(2008), 707-719.

[5] Al. Thagafi, M.A., Shahzad, N., Generalized I-non expansive selfmaps and invariant approximation, Acta. Math. Sin. (Engl. Ser.) 24(2008), 867-876.

[6] Al-Thagafi, M.A., Shahzad, N., A note on occasionally weakly compatible maps, Int. J. Math. Anal., 3(2) (2009) 55-58.

[7] Babu, G.V.R. and Alemayehu, G.N., A common fixed point theorem for weakly contractive mappings satisfying property (E.A.), Appl. Math. E-Note 10(2010), 167-174.

[8] Babu, G.V.R and Alemayehu, G.N., Points of coincidence and common fixed points of a pair of generalized weakly contractive mappings, J. Adv. Res. Pure Math., 2(2)(2010), 89-106.

[9] Babu, G.V.R. and Alemayehu, G.N., common fixed point theorems for occasionally weakly compatible maps satisfying property (E.A.) using an inequality involving quadratic terms, applied Mathematics Letters 24(2011), 975-981.

[10] Imdad, M., Ali, J. Jungcks common fixed point theorem and (E.A.) property, Acta Math. Sin (Engl. Ser.), 24(1) (2008), 87-94.

[11] Jungck, G., compatible mappings and common fixed points, Int. J. Math. Math. Sci. 9(4)(1986), 771-779.

[12] Jungck, G. and Rhoades, B.E., Fixed points for set valued functions without continuity, Indian J. Pure. Appl. Math. 29(3)(1998), 227-238.

[13] Jungck, G. and Rhoades, B.E., fixed point theorems for occasionally weakly compatible mappings, Fixed point theory, 7(2006), 287-296. 
[14] Liu, W., Wu, J. and Li, Z., Common fixed points of single valued and multivalued maps, Int. J math.math. Sci., 19(2005), 3045-3055.

[15] Murthy, P.P., Vara Prasad, K.N.V.V and Rashmi, Fixed points of nonlinear contraction, Adv. Fixed point Theory, 3 No. 4(2013), 600-607.

[16] Pathak, H.K., Rodriguez-Lopez and Verma, R.K., A common fixed point theorem using implicit relation and property (E.A.) in metric spaces, Filamat 21(2)(2007), 211-234.

[17] Sayyed S.A. and Sayyed, M., Some results of fixed points of nonlinear contraction, orient. J. phys.science Vol.2 (1)(2017).

[18] Tas. K., Telci, M. and Fisher, B., Common fixed point theorems for compatible mapping, Int. J. math. Math. Sci., 19(3)(1996), 451-456. 